\title{
Inventários biológicos rápidos de morcegos (Mammalia, Chiroptera) em três unidades de conservação do Amapá, Brasil
}

\author{
Ana C. M. Martins ${ }^{1}$; Enrico Bernard ${ }^{2} \&$ Renato Gregorin ${ }^{3}$
}

\author{
${ }^{1}$ Centro de Pesquisas Zôo-Botânicas e Geológicas, Instituto de Pesquisas Científicas e Tecnológicas do Amapá. Rodovia \\ Juscelino Kubitscheck, km 10, Distrito de Fazendinha, 68900-280 Macapá, Amapá, Brasil. \\ E-mail: ana.martins@iepa.ap.gov.br \\ ${ }^{2}$ Autor correspondente. Conservação Internacional. Avenida Governador José Malcher 652, 2a andar, 66035-100 Belém, \\ Pará, Brasil. E-mail: e.bernard@conservacao.org \\ ${ }^{3}$ Departamento de Biologia, Universidade Federal de Lavras. Caixa Postal 37, 37200-000 Lavras, Minas Gerais, Brasil. \\ E-mail: rgregorin@ufla.br
}

\begin{abstract}
Rapid biological surveys of bats (Mammalia, Chiroptera) in three conservation units in Amapá, Brazil. With a high biological diversity and good status of preservation, the northern part of the Amazon Basin, and specially the State of Amapá, have their fauna and flora poorly studied. Studies pointed that Amapá has several areas classified as highest priority for faunal inventories in the Amazon Basin, but even that few studies have been conducted. In order to fulfill part of these knowledge gaps, three of the most important conservation units in the State, namely the Tumucumaque Mountains National Park, the Amapá National Forest and the Rio Iratapuru Sustainable Development Reserve, were studied for different biological groups. The results of four bat surveys in these conservation units are presented here. With a sampling effort of 1730.5 mistnet.hours, 858 bats were recorded, belonging to 51 species, 36 genus and six families. At least 25 of these species are first records for the State, elevating to 73 the number of bat species already recorded in Amapá.

KEY WORDS. Amazon; Guyana Shield; mammals survey; Rapid Assessment Program (RAP); species distribution.

RESUMO. Com alta diversidade biológica e elevado grau de preservação, a porção norte da Bacia Amazônica, e em especial o Estado do Amapá, são ainda relativamente pouco conhecidos em relação à sua fauna e flora. Estudos apontam que o Amapá possui regiões classificadas como de alta ou muito alta importância para a realização de inventários de fauna na Amazônia Brasileira, havendo ainda grandes lacunas na realização destas amostragens. De forma a suprir parte destas necessidades, três das principais unidades de conservação do Estado, o Parque Nacional Montanhas do Tumucumaque, a Floresta Nacional do Amapá, e a Reserva de Desenvolvimento Sustentável do Rio Iratapuru, foram recentemente amostrados para diferentes grupos biológicos. São apresentados aqui os resultados de inventários de morcegos provenientes de quatro expedições a estas unidades. Após um esforço de 1730,5 rede.horas, quatro inventários registraram 858 capturas, de 51 espécies pertencentes a 36 gêneros e seis famílias. Destas, 25 espécies representam primeiras ocorrências para o Estado, elevando-se para 73 o número de espécies de morcegos registradas no Amapá.

PALAVRAS-CHAVE. Amazônia; distribuição de espécies; Escudo das Guianas; inventários de mamíferos; Programa de Avaliação Rápida (RAP).
\end{abstract}

Localizado no extremo norte do Brasil, o Amapá possui terras inseridas tanto no Escudo das Guianas quanto na Bacia Amazônica, o que pode colocá-lo como um importante local de estudo da fauna e flora do continente sul-americano. A diversidade de habitats no Estado inclui florestas de terra firme que vão desde regiões montanhosas ao norte até áreas mais baixas ao sul do Estado, além de florestas inundadas de várzea e igapó, complexos de lagos, extensas porções de mangue ao longo de sua costa, formações vegetais associadas a afloramentos rochosos, e uma porção significativa de cerrados amazônicos em sua área central (IBGE 2000). O Amapá possui mais de 90\% de sua superfície ainda não alterados por ações antrópicas (INPE 2006) e cerca de $56 \%$ de sua área sob proteção legal, na forma de um mosaico de 12 unidades de conservação, além de cinco Terras Indígenas. Todas estas características abrem importantes possibilidades de estudos da fauna eflora ainda preservados no Amapá. 
Mesmo apresentando alta diversidade biológica e elevado grau de preservação, a porção norte da Bacia Amazônica, incluindo o Escudo das Guinas, e em especial o Amapá, são relativamente pouco conhecidos em relação à sua fauna de mamíferos (Voss \& Emmons 1996). O Amapá possui regiões classificadas como de alta ou muito alta importância para a realização de inventários de mamíferos na Amazônia Brasileira, além de áreas que são insuficientemente conhecidas, mas consideradas de provável importância (Sılva et al. 2001). Esta situação é semelhante para outros grupos biológicos, como répteis, anfíbios e aves (OrEN 2001, Vogt et al. 2001, Azevedo-Ramos \& Gallati 2002).

Recentemente o Governo do Amapá adotou a proposta do Corredor de Biodiversidade do Amapá, que prevê a interligação de suas áreas protegidas, estudos sobre a viabilidade de criação de novas unidades e o estabel ecimento de um mosaico de sistemas de utilização sustentáveis nas áreas entre as unidades existentes. Para o sucesso do Corredor de Biodiversidade do Amapá, é necessário que estas unidades sejam efetivamente funcionais. Até o momento, nenhuma das unidades de conservação do Estado dispõe de plano de manejo e uma das lacunas identificadas para a elaboração destes planos é a carência de informações básicas sobre a diversidade biológica do Amapá. Poucos inventários biológicos já foram realizados no Estado, e em especial dentro de suas unidades de conservação, o que compromete diretamente o real conhecimento da diversidade biológica nestas unidades, prérequisito essencial para a elaboração de planos de manejo (IBAma 2003).

De forma a suprir parte destas necessidades, o Instituto de Pesquisas Científicas e Tecnológicas do Amapá (IEPA), a ONG Conservação Internacional (Cl-Brasil), o IBAMA Amapá e a Secretaria Estadual de Meio Ambiente do Amapá (SEMA) estabeleceram um programa para inventariar e mapear a ocorrência de espécies de grupos ecológicos representativos no Amapá, e em especial dentro de três das principais unidades de conservação do Estado, o Parque Nacional Montanhas do Tumucumaque (PNMT), a Floresta Nacional do Amapá (FNA), e a Reserva de Desenvolvimento Sustentável do Rio Iratapuru (RDSI). Este programa executou inventários rápidos da flora superior e da fauna de mamíferos, répteis, anfíbios, aves, peixes e crustáceos dentro e no entorno das unidades de conservação citadas, gerando conhecimento científico sobre a biodiversidade do Amapá e subsídios para a elaboração dos planos de manejo das unidades.

Nestes inventários, especial atenção foi dada aos quirópteros, uma vez que estes compreendem uma parcela significativa da fauna de mamíferos da região Neotropical e são freqüentementen egligenciados em inventários biológicos (Voss \& Emmons 1996, Emmons \& Feer 1997). O conhecimento sobre a real diversidade de espécies de morcegos existentes na região do Escudo das Guianas ainda é impreciso (LIM \& Norman 2002). Os sítios mais bem amostrados para morcegos na região oriental da Amazônia estão em I wokrama, na Guiana (LIM \& ENGSTROM 2001a, b), em Paracou, na Guiana Francesa (Simmons \& Voss 1998), na região de Belém (ERICA M. Sampaio, Universidade de
Ulm, comunicação pessoal), e Alter do Chão (Bernard \& Fenton 2002), ambas no Pará. A distribuição destes sítios mostra que não há uma continuidade nas amostragens entre as áreas nas Guianas e as do Brasil, representada exatamente por uma lacuna de amostragens no Amapá (mas veja PERACCHI et al. 1984). A fauna de morcegos do Estado é relativamente pouco conhecida e resultante na maioria das vezes de registros pontuais (ERICA M. SAmpaIo, Universidade de UIm, comunicação pessoal) em sua maior parte associados a estudos de caráter epidemiológico, principalmente ligados ao vírus da raiva, conduzidos por instituições estaduais e federais de saúde e agropecuária.

São apresentados aqui os registros de morcegos das quatro primeiras expedições do projeto de inventários biológicos realizados no Amapá. Estes registros buscam preencher uma lacuna de conhecimento que existe sobre a ocorrência e distribuição das espécies de morcegos presentes na porção oriental da Amazônia, e em especial no Amapá. Estes registros representam também as primeiras listas de espécies de morcegos para as três unidades de conservação amostradas e confirmam a primeira ocorrência de várias espécies no Estado.

\section{MATERIAL E MÉTODOS}

Os inventários biológicos realizados seguem o Programa de Avaliação Rápida (RAP) desenvolvido pela Conservation International (e.g. Atonso et al. 2001, Chermoff et al. 2001, MonTAMBAULt \& MISSA 2002), que consiste na execução de estudos de curta duração (2-4 semanas), focados no registro do maior número possível de espécies em grupos pré-determinados de organismos em áreas pouco conhecidas. O objetivo desta abordagem não é gerar uma lista completa de espécies para os locais visitados, mas sim fornecer dados primordiais sobre a diversidade de uma área, para que estes sejam disponibilizados rapidamente, seja para embasar futuros estudos ou para iniciativas emergenciais de conservação. Para este programa no Amapá foi estabelecido que em cada sítio visitado as amostragens deveriam ficar restritas a um círculo amostral de $3 \mathrm{~km}$ de raio e que cada grupo biológico receberia um esforço mínimo de 10 dias de inventário. A seleção dos sítios e do local exato a ser amostrado é feita com base em um mapeamento e geo-referenciamento das unidades de conservação, utilizando-se imagens de satélite para identificação das principais formações de vegetação. São usados ainda no processo de seleção dos sítios dados de relevo, hidrografia, solos, tipo e uso de recursos naturais, presença humana, e viabilidade de acesso. Idealmente, cada grande tipo de formação vegetacional identificado pelo processo de classificação das imagens deveria receber uma expedição de inventário biológico, mas para maximizar a diversidade de habitats amostrados, buscou-se priorizar áreas que pudessem conter mais de um ambiente, ou transições entre estes.

Os morcegos foram amostrados por meio do uso de redes de neblina, armadas no sub-bosque (até $3 \mathrm{~m}$ de altura), ao longo de trilhas previamente abertas no interior da floresta. A orientação destas trilhas foi feita de modo a cobrir a maior diver-

Revista Brasileira de Zoologia 23 (4): 1175-1184, dezembro 2006 
sidade possível de habitats. O esforço amostral foi calculado na forma de rede-horas, sendo que uma rede de 12 × 2,5 m aberta por uma hora é igual a uma rede.hora. O índice de capturas foi calculado dividindo-se o número de morcegos capturados pelo esforço amostral. Embora tenha-se buscado a padronização do esforço amostral, isso não foi possível, tendo este variado por noite e por expedição (Tab. I). Na maior parte das noites foram usadas 10 redes, abertas às 18:00 h. O horário de fechamento das redes variou entre noites e sítios (Tab. I). Em pelo menos uma noite em cada sítio amostrado, as redes foram abertas cruzando corpos d'água, como o Igarapé do Braço na FNA, ou as margens dos Rios Amapari e Mapaoni no PNMT, e Jari na RDSI. Na FNA, uma noite foi destinada a amostrar a área ao redor da sede do Ibama, localizada na confluência dos Rios Falsino e Araguari, onde havia uma colônia evidente de molossídeos que se abrigavam no telhado da construção. Nesta ocasião, utilizamos apenas três redes de $12 \times 2,5 \mathrm{~m}$, instaladas ao redor da casa, e abertas às 18:00 h, por apenas uma hora. Os dados de captura para esta noite foram computados separadamente.

A curva do coletor foi calculada para cada sítio amostrado, computando-se os esforços das noites amostradas em cada um deles e o acúmulo de espécies. O Índice Estimador de Diversidade de Chão: $S^{*}=$ Sobs + $\left(a^{2} / 2 b\right)$; onde $S^{*}$ é o número de espécies esperadas, Sobs é o número de espécies observado, a é o número de espécies registradas uma única vez, e b é o número de espécies registradas somente duas vezes, foi usado para estimar o número total de espécies esperadas para cada localidade.

A identificação dos exemplares capturados foi feita através de uma chave de identificação para morcegos da sub-região da Guiana (LıM \& ENGSTROM 2001a) edechaveainda não publicada para morcegos da Amazônia (Erica M. SAMPAIO, Universidade de UIm, comunicação pessoal). Muitas espécies foram identificadas mediante comparação direta com espécimes depositados no Museu de Zoologia da Universidade de São Paulo (MZUSP) e na Coleção de Morcegos do Laboratório de Chiroptera, no Departamento de Zoologia da Universidade Estadual Paulista de São José do Rio Preto (DZSJRP). A nomenclatura das espécies seguiu Koopman (1993), Lım (1997), que considera Artibeus planirostris
(Leach, 1821) como espécie válida para a região amostrada, Simmons \& Voss (1998) e Simmons (2005). Exemplares testemunhos das espécies registradas foram coletados e depositados na Coleção de Fauna do Amapá, sediada no Instituto de Pesquisas Científicas e Tecnológicas do Amapá (IEPA), Macapá.

O PNMT, a FNA e a RDSI são áreas prioritárias para a conservação da biodiversidade na Amazônia (CAPOBIANCo et al. 2001) eengl obam a maior parte dostipos representativos de fisionomias vegetais presentes no Estado, como as florestas de terra-firme e alagáveis, campinas e vegetação associada à afloramentos rochoSOS (IBGE 2000). Os principais rios do Amapá (e.g. Oiapoque, Araguari, Amapari e Jari) possuem suas nascentes dentro ou associadas a estas unidades de conservação. As características de cada unidade, a local ização precisa dos inventários e as datas de amostragem para morcegos são descritas a seguir.

FNA - Com uma área de aproximadamente 412.000 ha, a FNA localiza-se no centro do Estado (Fig. 1), situada entre os Rios Falsino e Araguari $\left(0^{\circ} 40^{\prime} \mathrm{N}, 51^{\circ} 10^{\prime} \mathrm{W} ; 2^{\circ} 50 \mathrm{~N}\right.$, 52 $\left.2^{\circ} 30^{\prime} \mathrm{W}\right)$, distante cerca de 120 km em linha reta da capital, Macapá. O acesso é por via fluvial, pelo Rio Araguari, a partir da cidade de Porto Grande, distante cerca de $30 \mathrm{~km}$ da entrada da unidade. A FNA apresenta uma vegetação predominantemente de florestas de terra-firme, com al gumas manchas menores de florestas al agadas, tabocais (bambus) e afloramentos rochosos. A altitude varia de 80 a 450 m acima do nível do mar. Esta unidade foi originalmente criada em 1989 para permitir a exploração madeireira, entretanto a complexidade topográfica e a dificuldade de acesso impediram até o momento que esta exploração seja feita em escala comercial dentro da unidade. O ponto amostrado localiza-se a $40 \mathrm{~km}$ acima da foz do Igarapé do Braço $\left(1^{\circ} 17^{\prime} 01^{\prime \prime} \mathrm{N}\right.$, $51^{\circ} 35^{\prime} 19^{\prime \prime} \mathrm{W}$ ), um afluente da margem direita do Rio Falsino (Fig. 1), e foi inventariado entre 04 e 15 de agosto de 2004.

PNMT - Criado em 2002, o Parque localiza-se no noroeste do Amapá (Fig. 1), tem uma área de cerca de 3.870.000 ha, e está inserido nos municípios de Pedra Branca do Amapari, Serra do Navio, Laranjal do Jari, Oiapoque e Calçoene, além de uma pequena faixa de terras em Almeirim, Pará. O PNMT faz fronteira com o Parque Indígena Tumucumaque, a Terra Indí-

Tabela I. Esforço amostral e índices de capturas de morcegos obtidos em quatro inventários biológicos rápidos na Floresta Nacional do Amapá (FNA), na Reserva de Desenvolvimento Sustentável do Rio Iratapuru (RDSI) e em duas expedições ao Parque Nacional M ontanhas do Tumucumaque (PNMT I e PNMT II), unidades de conservação do estado do Amapá, Brasil.

\begin{tabular}{lccccc}
\hline \multicolumn{1}{c}{ Locais } & FNA & PNMTI & PNMT II & RDSI & Total \\
\hline Noites de amostragem & 8 & 8 & 8 & 33 \\
Esforço (rede.horas) & 542 & 326 & 424 & 438,5 & 1730,5 \\
Capturas & 267 & 304 & 166 & 858 \\
Índice de capturas & $0,49(0-0,77)$ & $0,93(0,31-2,83)$ & $0,39(0,02-0,66)$ & $0,27(0,1-0,66)$ & 0,49 \\
Espécies & 35 & 29 & 27 & 121 & 51 \\
Espécies/captura & 0,13 & 0,09 & 0,16 & 0,15 & 0,06 \\
Espécies/esforço & 0,06 & 0,09 & 0,06 & 0,04 & 0,03 \\
Índice de Chao & 59 & 33 & 37 & 62 \\
\hline
\end{tabular}




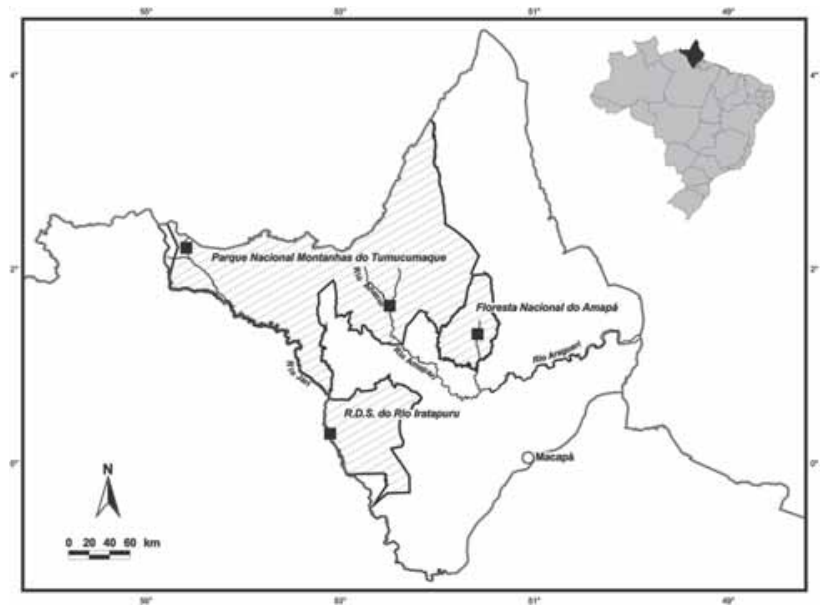

Figura 1. Localização dos inventários biológicos rápidos realizados no Parque Nacional Montanhas do Tumucumaque, na Floresta Nacional do Amapá e na Reserva de Desenvolvimento Sustentável do Rio Iratapuru, unidades de conservação do Estado do Amapá, Brasil.

gena Waiãpi e a FNA. Esta unidade de conservação é o maior parque de florestas tropicais contínuas do mundo e é acessível basicamente somente por via área ou por rios, em sua porção sul (Rio Amapari) ou na porção norte (Rio Oiapoque). O relevo é suavemente ondulado, com altitudes que variam de 100 a $400 \mathrm{~m}$ acima do nível do mar. Em sua porção norte, próximo à fronteira com o Suriname e a Guiana Francesa, existem diversos afloramentos de granito e insel bergs, que atingem até cerca de $800 \mathrm{~m}$ de altitude. A vegetação do Parque ainda não foi totalmente mapeada, mas predominam as florestas densas de terra firme, florestas de igapó, florestas de encosta e formações mais secas associadas aos afloramentos rochosos. O Parque recebeu duas expedições: na confluência dos Rios Amapari e Anacuí $\left(1^{\circ} 36^{\prime} 05^{\prime \prime} \mathrm{N}, 52^{\circ} 29^{\prime} 25^{\prime \prime} \mathrm{W}\right)$, inventariada entre 16 e 25 de setembro de 2004; e a segunda, entre 10 e 20 de janeiro de 2005, na porção norte, próximo à fronteira com o Suriname (211'36"N, 5435'15"W).

RDSI - Criada em 1997, com cerca de 806.000 ha, localiza-se no sudoeste do Amapá (Fig. 1), nos municípios de Laranjal do Jari, Mazagão e Pedra Branca do Amapari. O acesso se dá por barco, principalmente em sua porção sudoeste, através dos Rios Jari e Iratapuru. O relevo é suavemente ondulado e a altitude varia de 100 a 500 m acima do nível do mar. Há um predomínio de florestas de terra-firme, com grandes concentrações de castanheira-do-Brasil (Bertholletia excelsa Humb. \& Bonpl., 1808), sendo que existem populações tradicionais que vivem na porção sul da unidade e que exploram comercialmente este recurso. O local amostrado situa-se na margem esquerda do Rio Jari $\left(0^{\circ} 16^{\prime} 37^{\prime \prime} \mathrm{N}, 53^{\circ} 06^{\prime} 26^{\prime \prime} \mathrm{W}\right)$, cerca de $180 \mathrm{~km}$ em linha reta da sede do município de Laranjal do Jari e foi inventariado entre 12 e 20 de novembro de 2004.

\section{RESULTADOS}

FNA - O esforço amostral empregado neste inventário totalizou 542 rede.horas, resultando em mais de 267 capturas (Fig. 2) e no registro de 35 espécies de quirópteros em 27 gêneros (Tab. II). Dois indivíduos do gênero Peropteryx Peters, 1876 foram capturados, porém não incluídos na lista final de espécies devido à não identificação específica dos mesmos. Considerando-se este gênero, cinco das oito famílias de morcegos conhecidas para a Amazônia foram registradas: Phyllostomidae, Mormoopidae, Emballonuridae, Vespertilionidae e M olossidae. O número de capturas na FNA foi efetivamente maior, pois em três noites indivíduos de Artibeus planirostris, A. obscurus (Schinz, 1821) e Molossus molossus (Pallas, 1766) foram soltos após a captura e não tiveram seus dados registrados. Esta atitude foi tomada em função de que o elevado número de indivíduos destas espécies capturados simultaneamente estava dificultando o manuseio das redes e a captura de outras espécies. A. planirostris foi a espécie mais abundante, com 73 registros, seguido por C. perspicillata (Linnaeus, 1758), com 37 capturas, M. molossus, com mais de 25 capturas e A. obscurus, com 22. Quatorze das 35 espécies registradas foram representadas por apenas uma captura, e a família com maior número de espécies foi Phyllostomidae (30 espécies). O índice geral de capturas foi de 0,49 morcegos/rede.hora, computando-se apenas as capturas efetivamente registradas (267) e variou entre 0 e 0,77 por noite. As redes instaladas ao redor da sede do Ibama resultaram em um índice superior a 10 morcegos/rede.hora. O índice geral obtido foi de 0,13 espécie/captura e 0,06 espécie/rede.hora. A noite mais produtiva resultou em 23 espécies para um esforço de 115 rede.horas. O Índice de Chao calculado estima em 59 o número de espécies de morcegos para a localidade amostrada.

PNMT Expedição I - O esforço amostral foi de 326 rede.horas, resultando em 304 capturas (Fig. 2) e no registro de 29 espécies de morcegos, em 21 gêneros (Tab. II). Quatro famílias foram registradas: Phyllostomidae, Mormoopidae, Emballonuridae e Vespertilionidae. Artibeus planirostris foi a espécie mais abundante, com 157 capturas, seguido por $C$. perspicillata, com 29 capturas e A. obscurus, com 20. Oito das 29 espécies registradas foram representadas por apenas uma captura e 25 espécies pertenceram à família Phyllostomidae. O número de espécies registradas por noite variou entre seis e 16 . 0 índice de capturas geral foi de 0,93 morcegos/rede.hora, variando bastante entre noites, desde 0,31 até 2,83 morcegos/rede.hora. Esta expedição resultou em índices de 0,09 espécie/captura e 0,09 espécie/rede.hora. Segundo o Índice de Chao, o número esperado de espécies de morcegos para a localidade amostrada é 33.

PNMT Expedição II - Com um esforço amostral de 424 rede.hora foram capturados 166 morcegos (Fig. 2) pertencentes à 27 espécies, 19 gêneros e três famílias (Phyllostomidae, Mormoopidae e Noctilionidae). Artibeus planirostris foi a espécie mais abundante, com 77 capturas, seguido por C. perspicillata, Rhinophylla pumilio (Peters, 1865) e Lonchophylla thomasi J.A. Allen, 1904 (10 capturas cada), e Phyllostomus

Revista Brasileira de Zoologia 23 (4): 1175-1184, dezembro 2006 

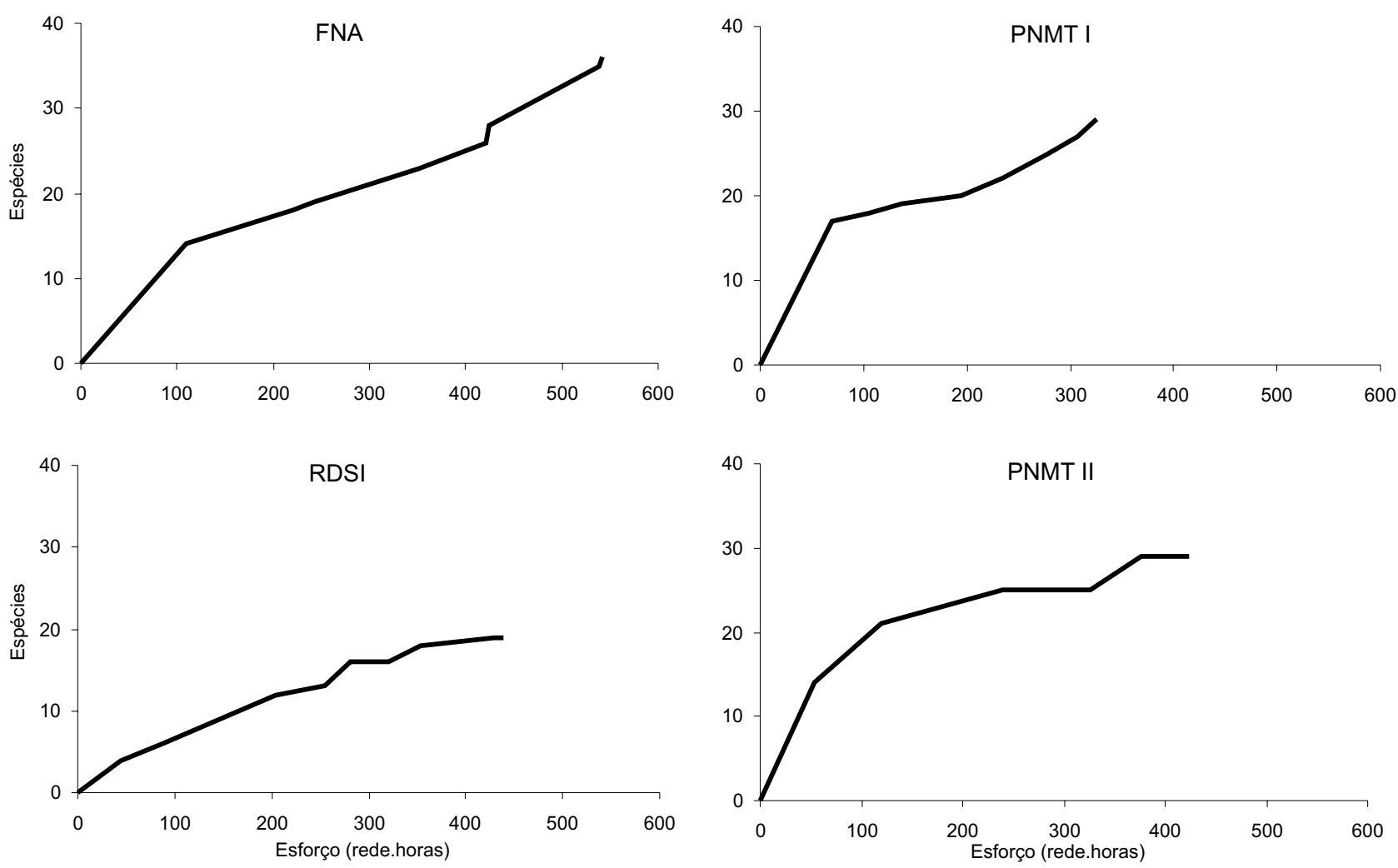

Figura 2. Curvas de acumulação de espécies baseadas no número de morcegos capturados em quatro expedições de inventário biológicos rápidos no Estado do Amapá, Brasil. (FNA) Floresta Nacional do Amapá, (RDSI) Reserva de Desenvolvimento Sustentável do Rio Iratapuru, (PNMT I) Expedição I, Parque Nacional Montanhas do Tumucumaque, (PNMT II) Expedição II, Parque Nacional Montanhas do Tumucumaque.

elongatus (E. Geoffroy, 1810), com nove (Tab. II). Dez das 27 espécies registradas foram representadas por apenas uma captura. O índice de capturas geral foi de 0,39 morcegos/rede.hora, variando por noite desde 0,02 até 0,66 morcegos/rede.hora. 0 número de espécies por noite de amostragem variou entre uma e 14. O índice de espécies/captura foi de 0,16 e o de espécie/ rede.hora 0,06. Para esta localidade são estimadas pelo índice de Chao 37 espécies.

RDSI - Com um esforço de 438,5 rede.horas foram capturados 121 morcegos (Fig. 2), pertencentes à 19 espécies, 13 gêneros e quatro famílias (Phyllostomidae, Emballonuridae, Molossidae e Vespertilionidae). Artibeus planirostris (37 capturas), A. lituratus (15) e R. pumilio (15) foram as espécies mais abundantes, enquanto outras quatro foram representadas por apenas uma captura. O número de espécies por noite de amostragem variou entre uma e 11 . O índice geral de capturas foi de 0,27 morcegos/rede.hora, variando de 0,10 a 0,66. Este inventário resultou em 0,15 espécie/captura e 0,04 espécie/ rede.hora. $\mathrm{O}$ índice de Chao estima 22 espécies para este sítio.

Quando computados conjuntamente, após um esforço de 1730,5 rede.horas, os quatro inventários registraram 858 capturas, de 51 espécies pertencentes a 36 gêneros e seis famílias (Emballonuridae, Mormoopidae, Phyllostomidae, Noctilionidae, Vespertilionidae e Molossidae). Quatorze espé cies foram representadas por apenas um indivíduo, sendo que seis foram registradas na FNA e cinco na segunda expedição ao Tumucumaque (Tab. II). Dez espécies foram registradas em todos os quatro inventários, e outras nove em apenas três. Artibeus planirostris respondeu por $40 \%$ do total de capturas. As outras três espécies mais abundantes foram, respectivamente, C. perspicillata $(8,8 \%)$, A. obscurus $(5,7 \%)$ e L. thomasi $(5,1 \%)$. Se cal culado o índice de Chao, 62 seria o total de espécies estimado para estes quatro inventários.

\section{DISCUSSÃO}

Previamente ao presente estudo, a fauna conhecida de morcegos do Estado do Amapá incluía 49 espécies (CARVALHo 1962, Piccinini 1974, Mok \& LACEy 1980, Peracchi et al. 1984, ERICA M. SAmPAIO, Universidade de UIm, comunicação pessoal). Os registros aqui apresentados acrescentam outras 25 espécies para esta lista, aumentando para 73 o número de espécies de morcegos registradas até o momento no Amapá. Para fins de comparação, 
Tabela II. Lista de espécies de morcegos para o Estado do Amapá, com dados de ocorrência obtidos em quatro inventários biológicos rápidos na Floresta Nacional do Amapá (FNA), na Reserva de Desenvolvimento Sustentável do Rio Iratapuru (RDSI) e em duas expedições ao Parque Nacional Montanhas do Tumucumaque (PNMT I e PNMT II). Na coluna "Total", espécies assinaladas com * indicam novas ocorrências para o Estado.

\begin{tabular}{|c|c|c|c|c|c|c|}
\hline Família & Espécie & FNA I & PNMTI & PNMT II & RDS I & Tota \\
\hline \multirow[t]{8}{*}{ Emballonuridae } & Centronycteris maximilliani (J. Fischer, 1829) & 1 & & & & $1 *$ \\
\hline & Diclidurus albus Wied-Neuwied, 1820 & & & & & \\
\hline & Diclidurus scutatus Peters, 1869 & & & & & \\
\hline & Peropteryx macrotis (Wagner, 1843) & & & & 7 & 7 \\
\hline & Rhinchonycteris naso (Wied-Neuwied, 1820) & 1 & 1 & & & 2 \\
\hline & Saccopteryx bilineata (Temminck, 1838) & & 1 & & & 1 \\
\hline & Saccopteryx canescens Thomas, 1901 & & & & & \\
\hline & Saccopteryx leptura (Schreber, 1774) & & & & & \\
\hline \multirow[t]{2}{*}{ Noctilionidae } & Noctilio albiventris Desmarest, 1818 & & & & & \\
\hline & N octilio leporinus (Linnaeus, 1758) & & & 1 & & 1 \\
\hline \multirow[t]{2}{*}{ Mormoopidae } & Pteronotus parnellii (Gray, 1843) & 22 & 1 & 2 & & $25^{*}$ \\
\hline & Pteronotus personatus (Wagner, 1843) & & & & & \\
\hline \multirow[t]{30}{*}{ Phyllostomidae } & Ametrida centurio Gray, 1847 & & & & & \\
\hline & Anoura caudifer (Geoffroy, 1818) & & 2 & & & 2 \\
\hline & Artibeus cinereus (Gervais, 1856) & 3 & 2 & 3 & 4 & 12 \\
\hline & Artibeus concolor Peters, 1865 & & 2 & & 1 & $3 *$ \\
\hline & Artibeus gnomus Handley, 1987 & & 1 & & 2 & $3 *$ \\
\hline & Artibeus lituratus (Olfers, 1818) & 4 & 5 & 2 & 15 & 26 \\
\hline & Artibeus obscurus (Schinz, 1821) & 22 & 20 & 4 & 3 & 49 \\
\hline & Artibeus planirostris (Leach, 1821) & 73 & 157 & 77 & 37 & 344 \\
\hline & Carollia brevicauda (Schinz, 1821) & 10 & 6 & 7 & 7 & $30 *$ \\
\hline & Carollia perspicillata (Linnaeus, 1758) & 37 & 29 & 10 & & 76 \\
\hline & Chiroderma trinitatum Goodwin, 1958 & 1 & & & & $1 *$ \\
\hline & Chiroderma villosum Peters, 1860 & & & 1 & & $1 *$ \\
\hline & Choeroniscus minor (Peters, 1868) & & 1 & & & $1 *$ \\
\hline & Chrotopterus auritus (Peters, 1856) & 2 & & 1 & 1 & $4 *$ \\
\hline & Desmodus rotundus (E. Geoffroy, 1810) & 1 & 1 & & & 2 \\
\hline & Diaemus youngi (Jentink, 1893) & & & & & \\
\hline & Diphylla ecaudata (Spix, 1823) & & & & & \\
\hline & Glossophaga soricina (Pallas, 1766) & 1 & & & & 1 \\
\hline & Lionycteris spurelli Thomas, 1913 & 1 & & 1 & & 2 \\
\hline & Lonchophylla thomasi J.A. Allen, 1904 & 15 & 11 & 10 & 8 & 44 \\
\hline & Lophostoma brasiliense Peters, 1866 & & & 2 & 2 & $4 *$ \\
\hline & Lophostoma schulzi (Genoways \& Williams, 1980) & & & 1 & & $1 *$ \\
\hline & Lophostoma silvicolum d'Orbigny, 1836 & 7 & 6 & & 4 & 17 \\
\hline & Macrophyllum macrophyllum (Schinz, 1821) & & & & 2 & 2 \\
\hline & Mesophylla macconnelli Thomas, 1901 & & & & & \\
\hline & M icronycteris megalotis (Gray, 1842) & & & & & \\
\hline & M icronycteris microtis Miller, 1898 & 3 & 2 & & & $5^{*}$ \\
\hline & M icronycteris minuta (Gervais, 1856) & 1 & & & & 1 \\
\hline & M icronycteris schmidtorum Sanborn, 1935 & & & 1 & & $1 *$ \\
\hline & M imon bennettii (Gray, 1838) & 2 & & & & $2 *$ \\
\hline
\end{tabular}

Revista Brasileira de Zoologia 23 (4): 1175-1184, dezembro 2006 
Tabela II. Continuação.

\begin{tabular}{|c|c|c|c|c|c|c|}
\hline Família & Espécie & FNA I & PNMTI & PNMT II & RDS I & Total \\
\hline & Mimon crenulatum (E. Geoffroy, 1803) & 2 & 4 & 1 & & 7 \\
\hline & Phylloderma stenops Peters, 1865 & & 1 & & & $1 *$ \\
\hline & Phyllostomus discolor Wagner, 1843 & 1 & 10 & 7 & & $18 *$ \\
\hline & Phyllostomus elongatus (E. Geoffroy, 1810) & 3 & 11 & 9 & 2 & 25 \\
\hline & Phyllostomus hastatus (Pallas, 1767) & 2 & 8 & 3 & & 13 \\
\hline & Platyrrhinus helleri (Peters, 1866) & 3 & 1 & 3 & & 7 \\
\hline & Platyrrhinus lineatus (Geoffroy, 1810) & & & 1 & & $1 *$ \\
\hline & Rhinophylla pumilio (Peters, 1865) & 6 & 8 & 10 & 15 & 39 \\
\hline & Sturnira lilium (E. Geoffroy, 1810) & & & & & \\
\hline & Sturnira tildae de la Torre, 1959 & 3 & 2 & 2 & 2 & $9 *$ \\
\hline & Tonatia saurophila Koopman \& Williams, 1951 & 6 & 3 & 1 & & $10 *$ \\
\hline & Trachops cirrhosus (Spix, 1823) & 1 & 4 & 3 & 1 & 9 \\
\hline & Trinycteris nicefori (Sanborn, 1949) & 4 & 2 & & & 6* \\
\hline & Uroderma bilobatum Peters, 1866 & 1 & & 2 & & 3 \\
\hline & Uroderma magnirostrum Davis, 1968 & & & & & \\
\hline & Vampyressa bidens (Dobson, 1878) & & & & & \\
\hline & Vampyressa thyone Thomas, 1909 & 1 & & & & $1 *$ \\
\hline & Vampyrodes caraccioli (Thomas, 1889) & 1 & & & & $1 *$ \\
\hline & Vampyrum spectrum (Linnaeus, 1758) & 1 & & 1 & & 2 \\
\hline Thyropteridae & Thyroptera tricolor Spix, 1823 & & & & & \\
\hline \multirow[t]{6}{*}{ Vespertilionidae } & Eptesicus brasiliensis (Desmarest, 1819) & & & & & \\
\hline & Eptesicus furinalis (d'Orbigny, 1847) & & & & & \\
\hline & Lasiurus blossevillii (Lesson and Garnot, 1826) & & & & & \\
\hline & Myotis albescens (Geoffroy, 1806) & 1 & & & 1 & 2* \\
\hline & Myotis nigricans (Schinz, 1821) & & & & & \\
\hline & Myotis riparia (Handley, 1960) & & 2 & & & 2* \\
\hline \multirow[t]{5}{*}{ Molossidae } & Eumops trumbulli (Thomas, 1901) & & & & & \\
\hline & Molossus molossus (Pallas, 1766) & 25 & & & & 25 \\
\hline & Molossus rufus E. Geoffroy, 1805 & & & & & \\
\hline & Nyctinomops laticaudatus (E. Geoffroy, 1805) & & & & 7 & $7 *$ \\
\hline & Promops nasutus (Spix, 1823) & & & & & \\
\hline Total & & 267 & 304 & 166 & 121 & 858 \\
\hline
\end{tabular}

nos dois Estados mais bem estudados para morcegos na Amazônia Brasileira (Pará e Amazonas) são conhecidas, respectivamente, 116 e 109 espécies (ErICA M. Sampalo, Universidade de UIm, comunicação pessoal ), e para a Guyana, Surinamee Guiana Francesa, países fronteiriços ou próximos ao Amapá, respectivamente, 121, 103 e 102 espécies (Lım \& EnGstrom 2001a). Tais números mostram o quanto a fauna de morcegos do Amapá é pouco conhecida e reforçam a necessidade de que mais estudos sejam conduzidos neste Estado que, potencialmente, pode conter uma das faunas mais ricas de morcegos da Amazônia Brasileira.

Entre as ocorrências mais importantes nos inventários, merecem destaque o primeiro registro formal de Mimon bennettii
(Gray, 1838) para a região Norte do país, a extensão da ocorrência de Vampyressa thyone Thomas, 1909, espécie até então registrada somenteno Acre, Amazonas eRondônia (Erica M. Sampaio, Universidade de UIm, comunicação pessoal, Valéria Tavares, American Museum of Natural Hisotry, comunicação pessoal), e o registro de Centronycteris maximilliani (J. Fischer, 1829), espécie rara em coleções. Outras espécies geral mente pouco comuns em inventários, como Vampyrum spectrum (Linnaeus, 1758), Chrotopterus auritus (Peters, 1856), Phyllostomus hastatus (Pallas, 1767), Lophostoma schulzi (Genoways \& Williams, 1980) e Phylloderma stenops Peters, 1865, também constam como registros significativos. 
Colônias de Rhinchonycteris naso (Wied-Neuwied, 1820), contendo de 5 a 15 indivíduos, são facilmente avistadas nos troncos ao longo das margens dos rios da região, entretanto poucos indivíduos desta espécie foram capturados com rede. Apenas um piscívoro foi capturado, talvez em função do fato de que a maior parte dos cursos d'água próximos dos locais de amostragem apresentar águas rápidas, com superfície movimentada, ambientenão ideal para estas espécies. A captura de morcegos hematófagos indica que os locais abrigam animais degrande porte, como antas, queixadas e catetos, fato confirmado pelos inventários de outros mamíferos conduzidos simultaneamente aos de morcegos (Claudia Silva, Instituto de Pesquisas Científicas e Tecnológicas do Amapá, comunicação pessoal) dominância de espécies frugívoras, como A. planirostris, A. obscurus e C. perspicillata, segue padrões esperados para amostragens na região Neotropical (e.g. Moreno \& Halffter 2000, Lim \& Engstrom 2001b, Bernard \& Fenton 2002, Sampaio et al. 2003).

Inventários de curta duração não são capazes de amostrar a total idade da fauna de morcegos de uma local idade e, de fato, as curvas do coletor indicam claramente que a diversidade de espécies de cada um dos sítios amostrados ainda não apresenta sinais de saturação (Fig. 2). Entretanto, a estrutura trófica das espécies registradas nos quatro inventários indica a presença de praticamente todas as guildas esperadas, desde frugívoros até grandes carnívoros, nectarívoros e hematófagos. Para inventários de espécies de morcegosna Amazônia, é esperado que se atinja 90\% das espécies do local somente após cerca de 1000 a 2700 capturas, um valor muito superior ao obtido em qualquer inventário rápido (BeRnARD \& FEnTON 2002). O baixo número de espécies insetívoras aéreas nas quatro expedições devese ao fato de que as amostragens não se estenderam ao dossel da floresta, o que certamente teria resultado no registro de algumas espécies desta guilda (BERNARD 2001).

A pluviosidadeesua sazonal idadena região amazônica têm uma forte influência sobre o número de capturas de morcegos e de espécies registradas (e.g. Bernard 2002, Bernard \& Fenton 2002). De fato, o inventário conduzido na RDSI, já na segunda metade de novembro, época seca na região, mesmo com o segundo maior esforço amostral, resultou no menor número de capturas (121) e de espécies (19) entre os inventários. Para fins de inventário, ou seja, do registro do maior número possível de táxons, a expedição à FNA foi a que rendeu o maior número de espécies. Entretanto, quando o esforço amostral empregado é computado, observa-se que a primeira expedição ao Tumucumaque foi a que apresentou a melhor relação entre espécie/ esforço, com um índice cerca de $50 \%$ maior do que o inventário na FNA. Tais diferenças indicam que a escolha de estações do ano mais favoráveis, noites sem luar, associados ao cuidado com a disposição das redes e o aumento da variedade de ambientes amostrados, podem ser estratégias que melhorem a representatividade das amostragens feitas em inventários rápidos.

Além de contribuírem para o melhor conhecimento da fauna de morcegos do Amapá, os resultados aqui apresentados constituem o primeiro estudo sobre a fauna de morcegos de unidades de conservação do Estado. O melhor conhecimento das espécies e principalmente de seu "status" de conservação pode contribuir significativamente para a elaboração de planos de manejo das unidades de conservação estudadas. Esta contribuição pode ser na forma da apresentação de listas de espécies que comprovem a alta biodiversidade presente nas unidades, justificando uma das principais razões de suas criações, a de preservação in situ da riqueza biológica. Tendo em vista que morcegos participam de importantes processos ecológicos, como a dispersão de sementes e a polinização de plantas, e interagem com um amplo espectro de outras espécies, agindo tanto como predadores quanto como presas (e.g. FLEMING 1988, Findley 1993, Fenton 2001), os dados de ocorrência de espécies aqui apresentados podem ser utilizados para se estabelecer parâmetros iniciais para a avaliação de impactos de atividades como a extração de madeira e de essências florestais, permitidas em unidades de uso direto como a FNA e a RDSI.

\section{AGRADECIMENTOS}

Ao então Diretor Presidente do Instituto de Pesquisas Científicas e Tecnológicas do Amapá, Antônio Carlos da Silva Farias, ao gerente Executivo do Ibama Amapá, Edivan Borges, e ao então Secretário Estadual de Meio Ambiente do Amampá, Edvaldo de Azevedo Souza, juntamente com suas equipes técnicas. Ao Exército Brasileiro, em especial ao Comandante do 340 Batalhão de Infantaria de Selva em Macapá, Ten. Cel. Moraes José Carvalho Lopes Jr, e ao Comandante do 4o Esquadrão de Aviação do Exército em Manaus, Ten. Cel. Paulo Roberto de Oliveira, à Força Aérea Brasileira, através do 10 Comando Aéreo Regional em Belém, e ao Corpo de Bombeiros da Polícia Militar do Amapá em Macapá. A Zilmo Amorim de Paiva e a todos os barqueiros, cozinheiros e assistentes que estiveram conosco durante as expedições. Aos curadores das coleções visitadas, Mario de Vivo (MZUSP) e Eliana Morielle-Versute (DZSJRP). Projeto financiado com recursos do Fundo Global para a Conservação, da Conservação Internacional, através de doação da Fundação Betty e Gordon Moore.

\section{REFERÊNCIAS BIBLIOGRÁFICAS}

Alonso, L.E.; A. Alonso; T.S. Schulemberg \& F. Dallmeier. 2001. Biological and social assessments of the Cordillera de Vilcabamba, Peru. Washington, Rapid Assessment Program Working Papers 12 and SI/MAB, Series 6, Conservation International, 298p.

Azevedo-Ramos, C. \& U. Galattı. 2002. Patterns of Amphibian Diversity in Brazilian Amazonia: Conservation Implications. Biological Conservation, Amsterdam, 103 (1): 103-111.

BERNARD, E. 2001. Vertical stratification of bat communities in primary forests of Central Amazon, Brazil. Journal of Tropical Ecology, Cambridge, 17 (1): 115-126.

BERNARD, E. 2002. Diet, activity and reproduction of bat species

Revista Brasileira de Zoologia 23 (4): 1175-1184, dezembro 2006 
(Mammalia, Chiroptera) in Central Amazonia, Brazil. Revista Brasileira de Zoologia, Curitiba, 19 (1): 173-188.

Bernard, E. \& M.B. Fenton. 2002. Species diversity of bats (Mammalia: Chiroptera) in forest fragments, primary forests, and savannas in central Amazonia, Brazil. Canadian Journal of Zoology, Ottawa, 80: 1124-1140.

Capobianco, J.P.R.; A. Veríssimo; A. Moreira; D. Sawyer; I. Santos \& L.P. PInTo. 2001. Biodiversidade na Amazônia Brasileira: avaliação e ações prioritárias para a conservação, uso sustentável e repartição de benefícios. São Paulo, Instituto Socioambiental, 540p.

Carvalho, C.T. 1962. Lista Preliminar dos Mamíferos do Amapá. Papéis Avulsos do Departamento de Zoologia de São PauIo, São Paulo, 15: 183-297.

Chermoff, B.; P.W. Willink \& J.R. Montambault. 2001. A biological assessment of the Rio Paraguay basin, Alto Paraguay, Paraguay. Rapid Assessment Program Bulletin of Biological Assessment, Washington, 19: 1-156.

Emmons, L.H. \& F. Feer. 1997. Neotropical Rainforest mammals: a field guide. Chicago, University of Chicago Press, 298p.

Fenton, M.B. 2001. Bats. Nova York, Facts on File, 222p.

FINDLEY, J.S. 1993. Bats: a community perspective. Cambridge, Cambridge University Press, 179p.

FLEMING, T.H. 1988. The short tailed fruit bat: a study in plantanimal interactions. Chicago, University Press, 365p.

IBGE. 2000. Atlas nacional do Brasil. Rio de Janeiro, Instituto Brasileiro de Geografia e Estatística, Diretoria de Geociências, 3a ed., 263p.

IBAMA. 2003. Roteiro metodológico para elaboração de plano de manejo para Florestas Nacionais. Brasília, Instituto Brasileiro do Meio Ambiente edos Recursos Naturais Renováveis, $56 \mathrm{p}$.

INPE. 2006. Monitoramento da Floresta Amazônica Brasileira por satélite Projeto Prodes. Instituto Nacional de Pesquisas Espaciais. Disponível na World Wide Web em: http:/ /www.obt.inpe.br/prodes [Acessado em XI.2005]

Koopman, K.F. 1993. Order Chiroptera, p. 137-241. In: D.E. WILSON \& D.M. REEDER (Eds). Mammals species of the world: a taxonomic and geographic reference. Washington, Smithsonian Institution Press, $2^{\text {nd }}$ Edition, 1312p.

LIM, B.K. 1997. Morphometric differentiation and species status of the allopatric fruit-eating bats Artibeus jamaicensis and A. planirostris in Venezuela. Studies on Neotropical Fauna and Environment, Amsterdam, 32: 65-71.

LIM, B.K. \& M.D. Engstrom. 2001a. Species diversity of bats (Mammalia: Chiroptera) in Iwokrama Forest, Guyana, and the Guianan subregion: implications for conservation. Biodiversity and Conservation, Berlin, 10: 613-657.

LIM, B.K. \& M.D. ENGSTROM. 2001b. Bat community structure at I wokrama Forest, Guyana. Journal of Tropical Ecology, Cambridge, 17: 647-665.

Lim, B.K. \& Z. Norman. 2002. Rapid Assessment of Small Mammals in the Eastern Kanuku Mountains, Lower Kwitaro
River Area, Guyana. A Biodiversity Assessment of the Eastern Kanuku Mountains, Lower Kwitaro River, Guyana. Rapid Assessment Program Bulletin of Biological Assessment, Washington, 26: 51-58.

MoK, W.Y. \& L.A. LACEY. 1980. Algumas considerações ecológicas sobre morcegos vampiros na epidemiologa da raiva humana na Bacia Amazônica. Acta Amazônica, Manaus, 10: 335-342.

Montambault, J.R. \& O. Missa. 2002. A Biodiversity Assessment of the Eastern Kanuku Mountains, Lower Kwitaro River, Guyana. Rapid Assessment Program Bulletin of Biological Assessment, Washington, 26: 1-88.

Moreno, C.E. \& G. Halffter. 2000. Assessing the completeness of bat biodiversity inventories using species accumulation curves. Journal of Applied Ecology, Oxford, 37: 149-158.

Oren, D.C. 2001. Biogeografia e conservação de aves na região amazônica, p. 97-109. In: J.P.R. CAPOBIANCo; A. Veríssimo; A. Moreira; D. SAWYer; I. SANTOS \& L.P. PINTO (Eds). Biodiversidade na Amazônia Brasileira: avaliação e ações prioritárias para a conservação, uso sustentável e repartição de benefícios. São Paulo, Instituto Socioambiental, 540p.

Peracchi, A.L.; S.D.L. Raimundo \& A.M. Tannure. 1984. Quirópteros do território Federal do Amapá, Brasil (Mammalia, Chiroptera). Arquivos da Universidade Federal Rural do Rio de Janeiro, Soropédica, 7 (2): 89-100.

PIccinını, R.S. 1974. Lista Provisória dos Quirópteros da Coleção do Museu Paraense Emílio Goeldi (Chiroptera). Boletim do Museu Goeldi, Nova Série, Zoologia, Belém, 11: 1-32.

Sampaio, E.M.; E.K.V. Kalko; E. Bernard; B. Rodriguez-Herrera \& C.O. HANDLEY. 2003. A biodiversity assessment of bats (Chiroptera) in a tropical lowland rainforest of Central Amazonia, including methodological and conservation considerations. Studies on Neotropical Fauna and Environment, Amsterdam, 38 (1):17-31.

Silva, M.N.F.; A.B. Rylands \& J.L. Patton. 2001. Biogeografia e conservação da mastofauna na floresta Amazônica Brasileira, p. 110-131. In: J.P.R. Capobianco; A. Veríssimo; A. Moreira; D. SAwyer; I. SAntos \& L.P. Pinto (Eds). Biodiversidade na Amazônia Brasileira: avaliação e ações prioritárias para a conservação, uso sustentável e repartição de benefícios. São Paulo, Instituto Socioambiental, 540p.

Simmons, N.B. 2005. Order Chiroptera, p. 312-525. In: D.E. WILSON \& D.M. REEDER (Eds). Mammal species of the world: a taxonomic and geographic reference. Baltimore, John Hopkins University Press, $3^{\text {rd }}$ ed., 2000p.

Simmons, N.B \& R.S. Voss. 1998. The mammals of Paracou French Guiana: a Neotropical lowland rainforest fauna. Part I. Bats. Bulletin of the American Museum of Natural History, New York, 237: 1-220.

Vogt, R.C.; G.M. Moreira \& A.C.O.C. Duarte 2001. Biodiversidade de répteis do bioma floresta amazônica e ações prioritárias para sua conservação, p. 89-96. In: J.P.R. CAPOBIANco; A. Veríssimo; A. Moreira; D. Sawyer; I. Santos \& L.P. Pinto

Revista Brasileira de Zoologia 23 (4): 1175-1184, dezembro 2006 
(Eds). Biodiversidade na Amazônia Brasileira: avaliação e ações prioritárias para a conservação, uso sustentável e repartição de benefícios. São Paulo, Instituto Socioambiental, 540p.

Recebido em 26.V.2006; aceito em 13.XI.2006.
Voss, R.S. \& L.H. Emmons. 1996. Mammalian diversity in Neotropical lowland rainforests: a preliminary assessment. Bulletin of the American Museum of Natural History, New York, 230: 1-115. 